\title{
IL LINGUAGGIO DELLA POLITICA \\ IL LESSICO DELLA POLITICA
}

Lo scopo del presente lavoro è di fornire un quadro sistematico della vasta problematica del lessico politico e di far luce su alcuni problemi lessicologici riguardanti la formazione delle parole e il prestito linguistico. L'articolo tratta del lessico politico in chiave lessicologica; la terminologia politica è innovativa e si arricchisce di nuove unità lessicali; i procedimenti principali che riguardano l'arricchimento del lessico politico sono la formazione delle parole, che crea i neologismi dal materiale già esistente nella lingua, e il prestito linguistico, che arricchisce la lingua dall'esterno di prestiti e forestierismi presi da altre lingue.

Keywords: linguaggio politico, lessico, lessicologia, formazione delle parole, neologismi, neologia, prestito linguistico, prestiti, forestierismi, calchi linguistici

\section{Introduzione}

La maggior parte dei termini del lessico politico derivano dal linguaggio comune, ma hanno assunto un significato tecnico e un valore tecnico nel linguaggio politico usato da teorici della politica nonché da coloro che adoperano i termini politici nella vita politica di ogni giorno.

Alcune parole fondamentali del lessico politico contemporaneo, politica e democrazia, ci sono state tramandate dagli scrittori greci. Il sostantivo politica è fondamentalmente la scienza che si occupa del potere. La politica, 'scienza e arte di governare lo Stato', 'scienza che si occupa del potere', è una parola dotta. ${ }^{1}$ La parola democrazia 'potere del popolo', 'governo del popolo'2 è arrivata in italiano attraverso il francese démocratie. La democrazia è etimologicamente il potere del popolo, il potere dei cittadini. La democrazia è sempre stata definita come il governo dei molti rispetto al governo di uno (monarchia), rispetto al governo di pochi (oligarchia) nonché rispetto alla mancanza di governo (anarchia). Le parole base, le parole-chiave del lessico politico contemporaneo - come politica, democrazia, potere, governo, popolo, nazione, libertà - aiutano a capire il significato di molti termini politici e il loro valore nell'ambito della scienza politica contemporanea.

Lo scopo di questo lavoro è di fornire un quadro sistematico della vasta problematica del lessico politico e di far luce su alcuni problemi lessicologici riguardanti la formazione delle parole e il prestito linguistico.

1 La parola politica non è arrivata in italiano per tradizione popolare, cioè non è arrivata per via diretta, ma è stata ripresa nel sec. XIII dal greco per via indiretta, attraverso i libri degli scrittori greci.

2 La parola democrazia è stata ripresa nel sec. XVI dal greco dēmokratía, parola composta di dêmos 'popolo' e kratia '-crazia'. 
Il lavoro tratta del lessico politico in chiave lessicologica. Il lessico della politica è innovativo, si arricchisce continuamente di nuove unità lessicali; i procedimenti principali che riguardano l'arricchimento del lessico politico sono prima di tutto i procedimenti della formazione delle parole, la quale crea i neologismi dal materiale già esistente nella lingua e arricchisce la lingua dall'interno. Il secondo procedimento importante che riguarda l'arricchimento lessicale è il prestito linguistico che arricchisce la lingua dall'esterno prendendo le parole intere da altre lingue.

Il materiale trattato in questo lavoro, senza pretesa alcuna di esaustività, riguarda il lessico della politica che è ricco, innovativo, creativo, moderno e si rinnova sempre.

\section{La formazione delle parole}

La lessicologia è una importante area di studi linguistici che si occupa della creatività lessicale; è la possibilità di arricchire il lessico di una lingua mediante formazioni ottenute con elementi già esistenti nella lingua, cioè mediante i procedimenti della formazione delle parole creando i neologismi che sono protagonisti dell'evoluzione linguistica. Per cui la formazione delle parole è la più importante fonte interna dell'arricchimento lessicale.

La neologia, che è l'insieme dei procedimenti che servono a formare parole nuove di una lingua, studia i neologismi, cioè le parole formate da altre parole italiane mediante la suffissazione, la prefissazione o la composizione delle parole.

\subsection{La suffissazione}

La suffissazione riguarda i nomi suffissati formati mediante i suffissi nominali denominali, deaggettivali e deverbali, gli aggettivi suffissati formati mediante i suffissi aggettivali denominali e deaggettivali e i verbi suffissati formati mediante i suffissi verbali denominali e deaggettivali.

\subsubsection{I nomi suffissati}

2.1.1.1. Il suffisso nominale deaggettivale -ità forma nomi astratti che indicano qualità o condizione. Il nome apartiticità 'caratteristica di chi/ciò che è apartitico', 'qualità di apartitico' deriva dall'aggettivo apartitico con il suffisso -ità; il nome democraticità 'caratteristica di chi/ciò che è democratico', 'qualità di democratico' ha come base l'aggettivo democratico; il nome governabilità 'caratteristica di ciò che è governabile', 'possibilità di governare un Paese in modo stabile' ha come base l'aggettivo governabile e politicità 'condizione/caratteristica di ciò che è politico' deriva dall'aggettivo politico.

2.1.1.2. Il suffisso nominale denominale e deaggettivale -ismo forma sostantivi astratti con un vasto ambito semantico; i suffissati in -ismo indicano movimenti politici, correnti politiche, dottrine politiche, tendenze di gruppi politici e sociali. I1 nome anarchismo 'atteggiamento anarchico', deriva dall'aggettivo anarchico; il nome 
bicameralismo indica sistema parlamentare in cui il potere legislativo è affidato a due Camere. $^{3}$

Il suffissato castrismo ha come base il nome dell'uomo politico cubano Fidel Castro e il suffissato bonapartismo ha come base il nome della famiglia Bonaparte. Il nome centrismo 'tendenza di gruppi politici a formare una coalizione di centro dalla quale siano escluse le destre e le sinistre' deriva da centro con il suffisso -ismo, colonialismo 'di colonia', 'relativo a colonia' deriva da coloniale, contrattualismo 'dottrina politica e giuridica dei sec. XVI-XVIII fondata sui principi del contratto sociale' deriva da contrattuale, correntismo 'tendenza dei partiti politici a dividersi a correnti' deriva da corrente 'movimento di massa'. Dal nome democratico si ha democraticismo 'comportamento di chi vuol sembrare democratico', da egemonia si ha egemonismo 'aspirazione, tendenza all'egemonia', da Europa si ha europeismo 'atteggiamento di chi è favorevole all'unità europea', 'movimento che mira a creare tale unità'; fascismo 'regime politico totalitario stabilito in Italia dal 1922 al 1943', 'ogni ideologia e regime politico fondato sul totalitarismo di destra' deriva da fascio, simbolo del partito; franchismo 'regime dittatoriale in Spagna' deriva dal nome del generale Francisco Franco e gandhismo 'insieme delle concezioni ideologiche dell'uomo politico M. K. Gandhi', 'movimento politico e sociale ispirato alle concezioni di Gandhi' deriva dal nome dell'uomo politico Gandhi. Dal nome integrazione si ha integrazionismo 'movimento o tendenza politica in favore dell'integrazione razziale', dal nome lega si ha leghismo 'fenomeno politico e sociale, diffuso spec. nell'Italia settentrionale, consistente nell'associarsi in leghe, e in particolare nella Lega Nord', dal nome di V. I. Lenin si ha leninismo 'la dottrina politica di Lenin' e dal nome di J. R. McCarthy si ha maccartismo 'atteggiamento di chi professa anticomunismo'. Il suffissato liberalismo è 'la dottrina e il movimento politico che si fonda essenzialmente sulla garanzia delle libertà individuali da parte dello Stato', machiavellismo è 'la corrente di pensiero politico che si ricollega alla dottrina di Machiavelli', maoismo è 'il pensiero e la pratica politica ispirati alle teorie marxiste di Mao Zedong', marxismo è 'l'insieme delle dottrine filosofiche, economiche e politiche elaborate da K. Marx e F. Engels, peronismo è 'il regime autoritario instaurato da Peron', stalinismo è 'la politica di Stalin' e Trotzkismo 'la dottrina comunista di Trotzky'.

I suffissati monocameralismo e unicameralismo sono 'sistemi parlamentari in cui il potere legislativo è affidato a una sola Camera'. Da partito si ha partitismo 'tendenza a risolvere i problemi del Paese nell'ambito dei partiti', da politico si ha politicismo 'tendenza a politicizzare tutto', da regionale si ha regionalismo 'tendenza politica favorevole alle autonomie regionali'. Il nome sindacalismo ${ }^{4}$ significa 'programma mirante a organizzare $i$ lavoratori in sindacati al fine di garantire gli interessi nei confronti di datori di lavoro'; il nome socialismo ${ }^{5}$ significa 'teoria e movimento politico-economico che

3 Il suffissato bicameralismo deriva da bicamerale sul modello dell'inglese bicameralism.

4 Il suffissato è formato dall'aggettivo sindacale e dal suffisso -ismo sul modello del francese syndicalisme.

5 Il suffissato è formato dall'aggettivo sociale e dal suffisso -ismo sul modello del francese socialisme e dell'inglese socialism. 
propugnava il possesso e il controllo dei mezzi di produzione da parte delle classi lavoratrici' e il nome totalitarismo 'regime politico in cui il potere viene concentrato nelle mani di un gruppo dominante' deriva dall'aggettivo totalitario.

2.1.1.3. Il suffisso nominale denominale e deaggettivale -ista forma sostantivi suffissati connessi con i suffissati in -ismo e indica attività politica, politica come professione; i suffissati in -ista hanno significato di 'seguace di una dottrina politica', 'fautore di un movimento politico', 'sostenitore di una corrente politica'.

I rispettivi suffissati non sono pochi: bonapartista 'chi segue o sostiene il bonapartismo', castrista 'fautore del castrismo', centrista 'chi appartiene al centro in senso politico', colonialista 'sostenitore/fautore del colonialismo', europeista 'sostenitore/ fautore dell'europeismo', fascista 'sostenitore/fautore del fascismo', franchista 'seguace/sostenitore di Franco o del franchismo', integrazionista 'chi sostiene l'integrazionismo', leghista 'chi aderisce al leghismo', 'militante/sostenitore di una lega, spec. della Lega Nord', leninista 'seguace del leninismo, maccartista 'sostenitore del maccartismo', machiavellista 'seguace del machiavellismo', 'studioso delle opere e del pensiero politico di Machiavelli', maoista 'seguace del maoismo, marxista 'seguace del marxismo', peronista 'seguace/fautore del peronismo', pluralista 'seguace del pluralismo', razzista 'fautore del razzismo', regionalista 'sostenitore del regionalismo', socialista 'chi si ispira al socialismo', ${ }^{6}$ stalinista 'fautore/sostenitore dello stalinismo', trotzkista 'seguace/sostenitore del trotzkismo'.

2.1.1.4. Il suffisso nominale deverbale -zione forma astratti deverbali che indicano azione politica, effetto e risultato dell'azione politica: balcanizzazione (da balcanizzare) 'il balcanizzare', 'il venire balcanizzato', comunistizzazione (da comunistizzare) 'il comunistizzare', 'il venire comunistizzato', democratizzazione (da democratizzare) 'il democratizzare', 'il democratizzarsi', egemonizzazione (da egemonizzare) 'l'egemonizzare', europeizzazione (da europeizzare) 'l'europeizzare', 'l'europeizzarsi', fascistizzazione (da fascistizzare) 'il fascistizzare', massificazione (da massificare), 'il massificare', politicizzazione (da politicizzare) 'il politicizzare', 'il politicizzarsi', sindacalizzazione (da sindacalizzare) 'il sindacalizzare', 'il venire sindacalizzato'.

La derivazione con suffisso 0 (zero) forma nomi deverbali: sciopero da scioperare.

\subsubsection{Gli aggettivi suffissati}

Qui rientrano anche gli aggettivi in -ista, come per esempio centrista 'che appartiene al centro in senso politico', colonialista 'che riguarda il colonialismo' che non saranno citati perché sono stati già elencati tra i nomi suffissati in -ista.

2.1.2.1. Il suffisso aggettivale denominale e deaggettivale -ario forma l'aggettivo dottrinario 'che si ispira esclusivamente ad una dottrina' il quale deriva da dottrina ${ }^{7}$ e l'aggettivo totalitario 'ispirato al totalitarismo' che deriva da totale e (autor)itario.

6 Il suffissato socialista deriva dall'aggettivo sociale e dal suffisso -ista sul modello del francese socialiste.

7 Il dottrinario in politica viene a indicare chi segue pedissequamente i principi di una dottrina politica; chi è portato a ragionare di dottrine piuttosto che ad affrontare in modo concreto i problemi. 
2.1.2.2. Il suffisso aggettivale denominale -iano forma aggettivi tratti prevalentemente da nomi propri o cognomi di famosi uomini politici e il significato dell'aggettivo suffissato comprende la loro dottrina politica, la loro opera politica, la loro attività politica: bismarckiano 'relativo al politico prussiano Bismarck', leniniano 'che si riferisce a V. I. Lenin e alla sua dottrina politica', machiavelliano 'proprio di $\mathrm{N}$. Machiavelli, del suo pensiero politico e delle sue opere', marxiano, 'che si riferisce al filosofo, economista e politico tedesco K. Marx', ${ }^{8}$ staliniano 'che si riferisce all'uomo politico e statista Stalin'.

2.1.2.3. Il suffisso aggettivale denominale -ico serve a formare aggettivi di qualità, di relazione e di rapporto. Dalla base anarchia si ha l'aggettivo suffissato anarchico 'proprio dell'anarchia', dalla base antisemita si ha l'aggettivo suffissato antisemitico 'relativo all'antisemitismo e agli antisemiti', da correntocrazia si ha correntocratico 'di correntocrazia, ispirato a correntocrazia' da Machiavelli si ha machiavellico 'relativo/ conforme alle politiche del Machiavelli'. Da massmediologia si ha massmediologico 'relativo ai mass media e alla massmediologia', e da mediologia, accorciamento di massmediologico, si ha mediologico; da meritocrazia si ha meritocratico 'basato sulla meritocrazia', da pacifista si ha pacifistico 'relativo al pacifismo e ai pacifisti', da partito si ha partitico 'relativo a uno o più partiti', da partitocrazia si ha partitocratico 'basato sulla partitocrazia', da politologia si ha politologico 'relativo alla politologia'.

2.1.2.4. Il suffisso agettivale denominale e deaggettivale -istico forma aggettivi connessi con i sostantivi in -ismo e in -ista; gli aggettivi suffissati indicano qualità, relazione e rapporto. L'aggettivo assolutistico 'dell'assolutismo' deriva da assolutismo o da assolutista, astensionistico 'relativo all'astensionismo' deriva da astensionismo, colonialistico 'pertinente al colonialismo' deriva da colonialismo o da colonialista, costituzionalistico 'relativo al costituzionalismo' da costituzionalismo, egemonistico ' $\mathrm{di} / \mathrm{re}-$ lativo all'egemonismo' da egemonismo. Gli aggettivi suffissati in -istico sono inoltre: estremistico 'proprio dell'estremismo e degli estremisti', europeistico 'proprio dell'europeismo e degli europeisti', federalistico 'del federalismo, dei federalisti, internazionalistico 'che riguarda l'internazionalismo', isolazionistico 'relativo all'isolazionismo e agli isolazionisti', leninistico 'relativo al leninismo e ai leninisti', liberalistico 'che concerne il liberalismo e i liberali', maoistico 'relativo al maoismo e ai maoisti', marxistico 'del marxismo e dei suoi seguaci', militaristico 'relativo al militarismo', 'di/da militarista', nazionalistico 'basato sul nazionalismo', 'proprio del nazionalismo', nazionalcomunistico 'relativo al nazionalcomunismo', nazionalsocialistico 'proprio del nazionalsocialismo', opportunistico 'proprio dell'opportunismo' o 'da opportunista', pluralistico 'relativo al pluralismo', populistico 'del populismo', 'da populista', razzisti-

8 L'aggettivo marxiano è adoperato con riferimento al pensiero filosofico, politico e economico di K. Marx, mentre gli aggettivi marxista e marxistico si riferiscono al marxismo. 
$c o$ 'del razzismo', 'da razzista', regionalistico 'da regionalista', 'conforme al regionalismo', sindacalistico 'che si riferisce al sindacalismo', totalitaristico 'concernente il totalitarismo', trasformistico 'concernente il trasformismo'.

2.1.2.5. Il suffisso -izio forma l'aggettivo correntizio 'relativo a una o più correnti di un partito politico' che deriva da corrente 'gruppo organizzato all'interno di un partito politico'.

\subsubsection{I verbi suffissati}

2.1.3.1. Il suffisso verbale -ificare forma il verbo massificare 'fare/rendere massa', 'portare su di uno stesso livello, eliminando così personalità e individualità'; deriva dal nome massa.

2.1.3.2. Il suffisso -izzare forma verbi da basi nominali e aggettivali come balcanizzare 'ridurre uno Stato nelle condizioni di disordine e di frammentazione in cui si trovavano gli Stati balcanici nei primi decenni del Novecento', comunistizzare 'rendere comunista', egemonizzare 'sottoporre alla propria egemonia', fascistizzare 'rendere fascista', liberalizzare 'rendere più libere le forze politiche e culturali', politicizzare 'imporre una finalità politica a qualunque $\cos { }^{\prime},{ }_{10}^{10}$ sindacalizzare 'organizzare in sindacato', 'rendere sensibile alle idee sindacali' (da sindacale), stalinizzare 'trattare secondo i metodi di Stalin'.

2.1.4. I neologismi di fresca data riguardanti la suffissazione nominale, aggettivale e verbale sono: bicameralismo, comunistizzare, europeistico, partitico, partitocratico, politicizzare, politizzazione, totalitarismo, stalinista, colonialistico, leninistico, liberalizzare, federalistico, pacifistico, razzistico, maccartismo, maccartista, regionalistico, estremistico, marxiano, marxistico, castrista, franchismo, integrazionismo, integrazionista, leniniano, massificare, massificazione, populistico, stalinizzare, correntismo, maoismo, maoista, balcanizzazione, cesaropapista, correntocratico, correntizio, sindacalizzare, massmediologico, mediologico, meritocratico, egemonistico, egemonizzare, europeizzazione, governabilità, egemonismo, astensionistico, egemonizzazzione, maoistico, politologico, leghismo.

\subsection{La prefissazione}

La prefissazione è propria dei nomi del lessico politico: con i prefissi $a$-, anti-, extra-, inter-, iper-, post-, sopra-, sotto-si ottengono i nomi prefissati; la prefissazione è propria anche degli aggettivi del lessico politico: con i prefissi $a$-, anti-, extra-, inter-,

9 Nel linguaggio politico sono molto frequenti gli avverbi di modo o maniera in -mente formati da aggettivi: assolutisticamente, democraticamente, egemonisticamente, estremisticamente, interpartiticamente, machiavellicamente, marxisticamente, meritocraticamente, militaristicamente, partitocraticamente, nazionalisticamente, pluralisticamente, politologicamente, populisticamente, razzisticamente, regionalisticamente, socialisticamente, trasformisticamente.

10

Il verbo suffissato politicizzare è formato dall'aggettivo politico sul modello dell'inglese to politicize. 
pre-, sopra- si ottengono gli aggettivi prefissati; però i prefissati verbali non sono numerosi.

\subsubsection{I nomi prefissati}

Il prefisso $a$-, detto anche $a$ o alfa privativo, indica mancanza, assenza relativamente a ciò che è espresso dall'aggettivo con cui forna il prefissato: apolitico composto da $a$-privativo e da politico significa 'chi è estraneo alla politica', 'chi manca di interesse per la politica'.

Il prefisso anti- con significato concettuale indica avversione, antagonismo, capacità o disposizione a contrastare: anticolonialismo 'avversione/opposizione al colonialismo o ai regimi coloniali', anticolonialista 'fautore/sostenitore dell'anticolonialismo', anticomunismo 'ostilità verso il comunismo', anticomunista 'chi è ostile al comunismo', antifascismo 'concezione e attività politica contraria al fascismo', antifascista 'chi si è opposto o si oppone al fascismo', antisemita 'chi è ostile nei confronti degli Ebrei'.

Il prefisso extra- con significato locale 'fuori' forma extraparlamentare 'chi aderisce a un movimento politico extraparlamentare', formato da extra- e parlamentare.

Il prefisso inter-con significato spaziale fa riferimento a posizione o condizione intermedia fra due cose o fra limiti di spazio: internazionalismo 'tendenza a favorire l'unione e la collaborazione fra gli Stati e i popoli di tutto il mondo' è formato dal prefisso inter-e dal nome nazionalismo; internazionalista formato dal prefisso inter- e dal nome nazionalista, 'chi favorisce l'internazionalismo' è formato dal prefisso intere dal nome nazionalista, il prefissato internazionalizzazione '1'internazionalizzare', 'l'internazionalizzarsi' è formato dal prefisso inter-e dal nome nazionalizzazione.

Il prefisso iper- significa 'sopra', 'oltre' 'esagerazione' e indica grado superiore al normale o eccessivo': iperpoliticismo formato dal prefisso iper- e dal nome politicismo 'tendenza esagerata a politicizzare troppo' formato dal prefisso iper-e dal nome politicismo.

Il prefisso post- con valore temporale significa 'posteriore', 'successivo', 'dopo' in postcomunismo o post-comunismo 'periodo successivo alla crisi dell'ideologia comunista a partire dalla fine degli anni Ottanta del Novecento', e in postcomunista 'seguace/sostenitore di un partito o dell'ideologia postcomunista'.

Il prefisso sopra-con significato concettuale indica superiorità in sopranazionalità 'autonomia e ampiezza dei poteri e funzioni di date organizzazioni internazionali rispetto agli Stati membri delle stesse', 'l'essere sopranazionale'.

Il prefisso sotto- con significato concettuale indica inferiorità quantitativa rispetto a.ciò che è normale in sottosviluppo 'condizione di arretratezza sociale e economica', formato dal prefisso sotto- e dal sostantivo sviluppo. 


\subsubsection{Gli aggettivi prefissati}

Gli aggettivi apartitico 'indipendente dai partiti politici' e apolitico 'che è estraneo alla politica o che manca di interesse per la politica' sono formati da $a$-privativo e da partitico e politico. ${ }^{11}$

I1 prefisso anti- forma l'aggettivo anticlericale ${ }^{12}$ 'che è contrario all'intervento del potere ecclesiastico nella vita politica', l'aggettivo antisemita 'che è ostile nei confronti degli Ebrei' e l'aggettivo antisistema 'contrario al sistema politico e sociale vigente'.

Il prefisso extra-forma l'aggettivo extraparlamentare 'che non fa parte dello schieramento dei partiti rappresentati in parlamento', 'che privilegia altre forme di lotta politica rispetto a quella parlamentare'; il prefissato è costituito dal prefisso extra- e dall'aggettivo parlamentare.

Il prefisso inter-forma l'aggettivo internazionale 'che concerne, interessa, collega più nazioni', ${ }^{13}$ l'aggettivo interparlamentare 'che concerne i due rami del Parlamento, cioè Camera e Senato' e l'aggettivo interpartitico 'relativo o comune a più partiti'.

Il prefisso pre- con significato temporale indica un rapporto di anteriorità nel tempo o un fatto che precede nel tempo come in premarxiano 'prima di Marx'; l'aggettivo sopranazionale 'che gode della sopranazionalità' è formato da sopra- e nazionale.

\subsubsection{I verbi prefissati}

I verbi prefissati e i verbi parasintetici non sono numerosi. Menzioniamo il verbo transitivo internazionalizzare 'rendere internazionale' formato dal prefisso inter-e da nazionalizzare, e il verbo intransitivo pronominale internazionalizzarsi 'assumere caratteristiche internazionali'.

Tra i parasintetici menzioniamo il verbo parasintetico spoliticizzare, formato dal prefisso $s$ - e dal suffisso verbale -izzare aggiunti simultaneamente all'aggettivo politico, 'rendere privo di caratteri politici' come in spoliticizzare il mondo, spoliticizzare i sindacati.

2.2.4. I neologismi di fresca data che riguardano la prefissazione nominale, aggettivale e verbale sono: sopranazionale/sovranazionale, sopranazionalità, spoliticizzare, internazionalizzare, sopranazionale/sovranazionale, interparlamentare, sottosviluppo, postcomunista, postcomunismo o post-comunismo, antisistema, iperpoliticismo, premarxiano.

11 Dall'aggettivo apartitico si forma anche l'avverbio di modo apartiticamente.

12 L'aggettivo anticlericale è formato dal prefisso anti-e dall'aggettivo clericale sul modello del francese anticlérical.

13

L'aggettivo internazionale è formato dal prefisso inter- e dall'aggettivo nazionale sul modello del francese international. 


\subsection{La composizione}

La composizione delle parole riguarda i nomi composti e gli aggettivi composti. I composti ottenuti con prefissoidi e suffissoidi e i composti ottenuti con elementi troncati sono formazioni molto interessanti dal punto di vista formativo e sono frequenti nel lessico politico contemporaneo.

\subsubsection{I nomi composti}

I nomi centrodestra (o centro-destra) 'alleanza politica tra partiti di centro e di destra' e centrosinistra (o centro-sinistra) 'alleanza politica tra partiti di centro e di sinistra' sono composti da centro e da destra, rispettivamente da sinistra.

Euro-, primo elemento di composizione che in parole composte della moderna terminologia politica significa 'europeo' entra in composizione con i nomi comunismo, comunista, destra, socialismo e socialista formando nomi composti eurocomunis$m o$ 'complesso delle posizioni politiche e teoriche tipiche di alcuni partiti comunisti occidentali negli anni '70 del Novecento', eurocomunista 'sostenitore/fautore dell'eurocomunismo', eurodestra 'insieme dei partiti politici europei di sinistra', eurosocialismo 'insieme delle comunanze ideologiche, politiche, storiche e istituzionali tra i partiti socialisti, socialdemocratici e laburisti dell'Europa occidentale' e eurosocialista 'sostenitore/fautore dell'eurosocialismo'.

Liberale e socialista formano il composto liberalsocialista 'fautore/seguace/sostenitore del liberalsocialismo'. Nazionale entra in composizione con socialista, comunismo e comunista formando i composti nazionalsocialista 'aderente al nazionalsocialismo', nazionalcomunismo 'tendenza ad adeguare il comunismo a esigenze e situazioni nazionali' e nazionalcomunista 'sostenitore/seguace del nazionalcomunismo'. Non allineamento (o non-allineamento) è 'condizione dei paesi non allineati'. Sociale entra in composizione con democratico e con democrazia formando i composti socialdemocratico $^{14}$ 'chi segue la socialdemocrazia', 'relativo o appartenente al Partito Socialista Democratico Italiano fondato nel 1947' e socialdemocrazia' 15 'socialismo di tipo riformista che rifiuta i metodi rivoluzionari'.

\subsubsection{Gli aggettivi composti}

Euro- entra in composizione con comunista e con socialista formando i composti eurocomunista 'relativo all'eurocomunismo' e eurosocialista 'relativo all'eurosocialismo'. Liberale e nazionale entrano in composizione con socialista formando liberalsocialista 'relativo al liberalsocialismo' e nazionalsocialista 'nazionalsocialistico'. Il composto non allineato si riferisce a Paesi che non aderiscono né al blocco occidentale né a quello orientale.

14 L'aggettivo composto socialdemocratico è formato dall'aggettivo sociale e dall'aggettivo democratico sul modello del tedesco Sozialdemokratisch.

15 Il nome composto socialdemocrazia è formato dall'aggettivo sociale e dal nome democrazia sul modello del tedesco Sozialdemokratie. 
Il composto socialdemocratico ${ }^{16}$ significa 'che segue la socialdemocrazia', 'relativo o appartenente al Partito Socialista Democratico Italiano fondato nel 1947'; 'della socialdemocrazia'.

\subsubsection{I composti ottenuti con elementi colti o dotti}

L'apporto che il greco e il latino danno alla formazione di nuove parole italiane attraverso affissi neoclassici - prefissoidi e suffissoidi - detti anche elementi di composizione, è molto importante. A partire dal Settecento l'influsso del latino e del greco è molto forte sul linguaggio scientifico. Numerose parole composte sono costituite da elementi lessicali latini e greci; i prefissoidi e i suffissoidi derivati dal latino e dal greco sono numerosi, sono numerosi soprattutto quelli derivati dal greco: auto-, bi-, demo-, geo-, mono-, neo-, oligo-, pluri-, poli-, ultra-, uni-, tele-, video-, -crazia, -logia, -logo.

\subsubsection{I prefissoidi}

Il prefissoide auto- con significato 'di sé stesso' o 'da sé' forma il composto autocritica 'critica di sé stessi e delle proprie azioni', e in paesi o partiti comunisti il composto assume un nuovo significato 'riconoscimento pubblico, spesso imposto, dei propri errori politici'; il composto autodeterminazione ${ }^{17}$ è 'autodeterminazione dei popoli', e 'il diritto di ciascun popolo di scegliere autonomamente la propria condizione politica'; il composto autogoverno si riferisce alle ex-colonie, è 'autogoverno dei popoli concesso ai paesi che raggiungono l'indipendenza'.

$B i$-, primo elemento di composizione 'due', 'due volte', 'composto di due', entra in composizione con camerale formando bicamerale 'sistema parlamentare costituito di due camere legistative' 18 .

Demo-, tratto da democrazia, in parole composte della moderna terminologia politica significa 'democratico'; entra in composizione con cristiano formando democristiano, aggettivo e nome, 'della Democrazia Cristiana', 'iscritto alla Democrazia Cristiana/sostenitore della Democrazia Cristiana' e con plutocrazia formando demoplutocrazia, 'nella pubblicistica fascista regime solo formalmente democratico', sul tipo formativo di democrazia.

Geo-, accorciamento di geografia 'considerato dal punto di vista geografico', entra in composizione con il nome politica formando il composto geopolitica 'scienza che studia le basi e le ragioni geografiche dei problemi politici ed economici' e con l'aggettivo politico forma il composto geopolitico 'della geopolitica', 'relativo alla geopolitica'.

L'aggettivo composto socialdemocratico è formato dall'aggettivo sociale e dall'aggettivo democratico sul modello del tedesco Sozialdemokratisch.

17 Il composto autodeterminazione è formato dal prefissoide auto-e dal nome determinazione sul modello dell'inglese self-determination.

18 Il composto bicamerale è formato dal prefissoide $b i$ - e dall'aggettivo camerale secondo il modello inglese bicameral. 
Mono-, primo elemento di composizione che significa 'uno', 'uno solo', 'costituito da uno solo', forma il composto monocamerale 'che si riferisce al sistema parlamentare basato su una sola camera legislativa'.

$\mathrm{Neo}$-, primo elemento di composizione che significa 'nuovo', 'recente', 'moderno', entra in composizione con il nome fascismo formando il composto neofascismo 'movimento politico che, dopo il 1945, ha ripreso le dottrine e le finalità politiche del fascismo' e con il nome fascista forma il composto neofascista 'aderente al neofascismo'. Neo- entra in composizione anche con il nome ghibellinismo formando il composto neoghibellinismo 'movimento ideologico e politico che, durante il Risorgimento, si contrappose al neoguelfismo' e con l'aggettivo ghibellino forma l'aggettivo e il nome neoghibellino 'del neoghibellismo', 'fautore/seguace del neoghibellismo'. Da neo- e dal nome guelfismo si forma il composto neoguelfismo 'movimento politico, sostenitore di un rinnovamento democratico del cattolicesimo' e da neo-e dal nome guelfo si formano l'aggettivo e il nome neoguelfo 'del neoguelfismo', 'fautore/ seguace del neoguelfismo'.

Pluri-, primo elemento che significa 'in numero maggiore di uno', entra in composizione con l'aggettivo partitico formando il composto pluripartitico 'che concerne più partiti'.

Ultra- fa riferimento a una qualità o condizione che supera la norma, quindi indica eccesso; entra in composizione con il nome destra e forma il composto ultradestra 'insieme di partiti o gruppi politici che operano nell'area dell'estrema destra'; e con il nome sinistra forma il composto ultrasinistra 'insieme di gruppi politici che si organizzano e operano nell'area della sinistra extraparlamentare e alla sinistra di quella istituzionale'.

Il primo elemento uni-, che fa riferimento a una sola unità, entra in composizione con l'aggettivo camerale formando l'aggettivo composto unicamerale 'che si riferisce al sistema parlamentare basato su una sola camera legislativa'. ${ }^{19}$

Video-, primo elemento in parole composte del linguaggio scientifico e tecnico che hanno riferimento con la vista o con la televisione, entra in composizione con l'aggettivo plasmato formando il composto videoplasmato 'che si forma con la vista, con l'esempio, l'insegnamento, l'educazione, che si forma secondo il modello'. Il prefissoide video- entra in composizione anche con i nomi politica e potere formando i composti videopolitica e videopotere. I due composti sono sinonimi, significano 'potere rappresentato dalla televisione che ha la capacità di condizionare le opinioni del pubblico televisivo'. ${ }^{20}$

19 Il composto unicamerale è formato secondo il modello inglese unicameral.

20 Video- è un elemento lessicale latino, cioè un elemento di composizione derivato dal latino, ma entrato in italiano attraverso la lingua inglese. 


\subsubsection{I suffissoidi}

Molto spesso i composti sono costituiti da due elementi di composizione colti o dotti, dal prefissoide e dal suffissoide.

Il secondo elemento di composizione -archia che significa 'governo', 'dominio' si trova nei seguenti composti dotti: monarchia 'governo di uno', nome formato da mono- e -archia; oligarchia 'governo di pochi', nome formato da oligo- che significa 'poco, pochi' e -archia; poliarchia 'governo di molti', nome formato da poli-che indica molteplicità numerica o quantitativa e da -archia.

Il suffissoide -crazia che significa 'potere, dominio' entra in composizione con il nome corrente 'gruppo organizzato all'interno di un partito politico' formando il composto correntocrazia 'il potere esercitato dalle correnti in seno ai partiti politici'; con demo-, primo elemento che in parole dotte composte significa 'popolo', forma il composto democrazia. Da merito e da -crazia si forma il composto meritocrazia 'concezione per cui ogni forma di riconoscimento come ricchezza, successo negli studi o nel lavoro, è esclusivamente commisurata al merito individuale'; da partito e da -crazia si forma partitocrazia, 'governo dei partiti'. Il composto tecnocrazia significa 'potere dei tecnici nella vita dello stato'.21

Il suffissoide -crazia entra in composizione con il prefissoide tele-, accorciamento di televisione, formando il composto telecrazia 'potere rappresentato dal mezzo televisivo per la sua capacità di condizionare le opinioni degli spettatori', ed entra in composizione con il prefissoide video-formando il composto videocrazia, che è sinonimo di telecrazia.

Il suffissoide -logia entra in composizione con il nome Cremlino formando il composto cremlinologia 'analisi della politica dell'Unione Sovietica o, dopo il 1991, della Russia', e il suffissoide -logo 'scienza' forma cremlinologo, 'esperto di cremlinologia, esperto della politica del governo sovietico che ha sede nel Cremlino'. Il nome mass media e-logia formano massmediologia 'studio dei mass media' e il nome media e-logia formano mediologia. Il nome mass media e -logo formano massmediologo 'studioso/esperto di massmediologia' come pure il nome media e -logo formano mediologo.

Il suffissoide -oide forma parole composte di formazione moderna; indica affinità, inclinazione: dal nome fascista e da -oide si ha fascistoide 'chi/che mostra affinità/inclinazione al fascismo' (spregiativo); da liberale e -oide si ha liberaloide 'chi/che è favorevole al liberalismo, in modo superficiale e approssimativo'; da socialista e -oide si ha socialistoide.

\subsubsection{I composti con elementi troncati}

La composizione delle parole è caratterizzata da nuovi tipi di composti e da nuovi procedimenti formativi. Ciò si riferisce prima di tutto ai composti ottenuti con ele-

21 Il composto tecnocrazia è formato da due elementi dotti; dal prefissoide tecno- che significa 'capacita tecnica' e fa riferimento a specializzazione tecnica e dal suffissoide -crazia sul modelo dell'inglese tecnocracy. 
menti troncati. Questo tipo particolare di composti è proprio dell'italiano contemporaneo.

Negli esempi che seguono il primo elemento di composizine è troncato. La parola anarcoide 'propenso all'anarchia' è un neologismo composto da anarc(hico) e -oide; cesaropapismo 'sistema di relazione fra potere politico e potere religioso' è composto da cesar(ismo) e papismo; liberalsocialismo 'dottrina che vuole conciliare i principi politici del liberalismo con i principi sociali del socialismo' è composto da liberal(ismo) e socialismo; plutodemocrazia 'demoplutocrazia' si ottiene con pluto(crazia) e dernocrazia; politologia 'disciplina che studia i sistemi politici' si ottiene con polit(ica) e -logia; socialcomunista 'che/chi partecipa a un'alleanza costituita dal PCI e dal PSI' è formato da social(ista) e comunista.

2.3.4. I neologismi di fresca data che si riferiscono alla composizione delle parole nel linguaggio politico sono: bicamerale, partitocrazia, unicamerale, nazionalcomunismo, democristiano, non allineamento, cremlinologo, non allineato, policentrismo, correntocrazia, telecrazia, centrodestra, cremlinologia, meritocrazia, politologo, ultradestra, ultrasinistra, eurocomunismo, eurocomunista, eurosocialista, politologia, massmediologo, monocamerale, eurosocialismo, mediologico, nazionalcomunista, eurodestra, mediologo, mediologia, videocrazia, videoplasmato, videopolitica, videopotere.

\section{Le unità lessicali superiori}

La composizione delle parole nell'italiano d'oggi è caratterizzata da un tipo particolare di composti: le unità lessicali superiori, unità composte di più elementi. Oltre a questo termine in linguistica si sono coniati altri termini: collocazioni ristrette, lessemi complessi, parole giustapposte, sintagmi lessicalizzati. ${ }^{22}$

Le unità lessicali superiori sono molto frequenti nell'italiano contemporaneo e riguardano anche il linguaggio della politica. Per quanto riguarda la loro forma si distinguono più tipi di unità lessicali superiori:

\subsection{Nome + prep di + Nome}

attività di spionaggio, autodeterminazione dei popoli, autonomizzazione della politica, colpo di stato, crimini di guerra, diritto di proprietà, diritto di cittadinanza, diritto di resistenza, diritti dell'uomo, divisione dei poteri, estremismo di destra, estremismo di sinistra, fenomeni di massificazione, gruppi d'interesse, gruppi di pressione, guerra dei nervi, lessico della politica, libertà di scelta politica, libertà di parola, Paesi del Terzo mondo,

22 Cfr. C. Marello, 1996, pp. 40-41: “...ci interessano quei sintagmi formati da più parole che si comportano come se fossero una parola sola. Vengono detti sintagmi lessicalizzati, perché la lessicalizzazione è appunto quel procedimento per cui i rapporti sintattici fra parole si irrigidiscono, la combinazione da libera diventa fissa e il sintagma viene considerato alla stregua di un'unità del lessico. Gran parte dei procedimenti di composizione nel lessico italiano contemporaneo si esplica attraverso la giustapposizione di parole, più che attraverso l'unione grafica dei componenti". 
politica di accerchiamento, politica d'isolamento, politico di professione, professionalizzazione della politica, Ragion di Stato, separazione dei poteri, servizi di sicurezza, sistemi di partito, società di massa, stato del benessere, Stato di polizia, tendenza di destra, tendenza di sinistra.

\subsection{Nome + Nome}

conferenza stampa, guerra lampo, notizia lampo, marxismo-leninismo, marxista-leninista, parola-chiave.

\subsection{Nome + Aggettivo (Aggettivo + Nome)}

comportamento elettorale, comunicazione politica, coalizione governativa, corruzione politica, cultura politica, democrazia costituzionale, democrazia diretta, democrazia indiretta, democrazia rappresentativa, democrazia parlamentare, democrazia politica, democrazie popolari, eguaglianza politica, guerra fredda, forze armate, fronte popolare, integrazione razziale, libertà politiche, potere esecutivo, opinione pubblica, opportunismo politico, ordine pubblico, Paesi non allineati, Paesi non impegnati, Paesi sottosviluppati, partecipazione politica, partiti politici, persuasione politica, politica videoplasmata, potere politico, professionalismo politico, propaganda politica, questione meridionale, rappressaglia pacifica, rappressaglia armata, rappresentanza politica, regime politico, sciopero generale, separatismo politico, sistema politico, sistema elettorale, socialismo democratico, socialismo reale, socialismo riformista, socialismo rivoluzionario, socializzazione politica, terrorismo politico, uomo politico.

\section{Sigle}

Le sigle godono di una particolare fortuna: sono espressioni sintetiche, semplici ed economiche. L'italiano contemporaneo ha tendenza a coniare le sigle e in particolare il lessico politico ha tendenza a usare le sigle che indicano movimenti politici, gruppi politici, partiti politici, club politici, associazioni, organizzazioni politiche. Ecco l'elenco delle principali sigle che riflettono la vita politica di un paese:

ACPOL - Associazione di Cultura POLitica; AD - Alleanza democratica; AN Alleanza Nazionale/Alleato nazionale; ANFI - Associazione Nazionale Forza Italia (club); AV - Avanguardia Operaia; CCD - Centro Cristiano Democratico; CDR - Cristiano Democratici per la Repubblica; CDU - Cristiano Democratici Uniti; CISP - Centro Internazionale Studi Politici; DC - Democrazia Cristiana; DP - Democrazia Proletaria; DS Democratici di Sinistra; F. d. S. - Federazione dei Socialisti; FI - Forza Italia; FLD Federalista LiberalDemocratico (gruppo); GIP - Gruppi di Impegno Politico; MLS Movimento dei Lavoratori per il Socialismo; MSI-DN - Movimento Sociale Italiano Destra Nazionale; PCI - Partito Comunista Italiano; P d'A - Partito d'Azione; PDS Partito Democratico della Sinistra; PdUP - Partito di Unità Proletaria per il comunismo; PRC - Partito di Rifondazione Comunista o RC - Rifondazione Comunista; PRI - Partito Repubblicano Italiano; PSI - Partito Socialista Italiano; RS - Rinascita Socialista; 
SDI - Socialisti Democratici Italiani; U.d.C. - Unione di Centro; UDeuR - Unione dei Democra-tici per l'Europa; UDR - Unione dei Democratici per la Repubblica o Unione Democratica per la Repubblica.

\section{Il Prestito linguistico}

Il prestito linguistico arricchisce la lingua di nuove unità lessicali: per prestiti s'intendono parole prese da una lingua straniera e per forestierismi s'intendono parole entrate in una lingua in tempi recenti; quindi i forestierismi si riferiscono alle lingue moderne, non alle lingue classiche. L'importanza dei latinismi e dei grecismi nella formazione del lessico italiano è grandissima; sono entrati nella lingua comune ed hanno arricchito i linguaggi tecnico-scientifici, e in particolare il linguaggio politico che aveva bisogno di nuovi termini. I latinismi e i grecismi hanno una storia interrotta; sono parole dotte che sono state riprese dai testi latini e greci ad opera di persone colte e sono state introdotte nella lingua italiana. A partire dall'Umanesimo in poi gli scienziati europei si rivolgevano al latino e al greco per formare i termini dei linguaggi tecnico-scientifici. Soprattutto a partire dal Settecento, i latinismi e i grecismi entrano in italiano dal francese e dall'inglese; sono parole che riguardano vari settori della scienza e in particolare il settore della politica.

5.1. Ricordiamo nel Duecento il latinismo opposizione, dal latino tardo oppositio$n e(m)$, parola dotta che significa 'insieme delle forze politiche contrarie al Governo'; nel Trecento il latinismo resistenza, dal latino tardo resistěntia $(m)$, parola dotta che significa 'sforzo tendente a opporsi, a resistere all'azione di qualcuno e di qualcosa' e la parola dotta rivoluzione, dal latino tardo revolutione $(m)$, 'violento e profondo rivolgimento dell'ordine politico-sociale' che in accezione politica è un calco sul francese révolution. Ricordiamo nel Cinquecento il francesismo alleanza, dal francese alliance, 'alleanza tra Stati per comunanza di interessi' e il grecismo aristocrazia, dal greco aristokratia, 'potere dei migliori' 'governo dei migliori'.

5.2. Nel Seicento troviamo i grecismi anarchia, dal greco anarchia, 'mancanza di governo', l'aggettivo aristocratico, dal greco aristokraticós, 'che appartiene all'aristocrazia', l'aggettivo monarchico, dal greco monarchikós, 'della monarchia', l'aggettivo oligarchico, dal greco oligarchikós, 'di oligarchia', 'proprio dell'oligarchia' e il grecismo democratico, dal greco demokratikós 'della democrazia', 'che professa i principi della democrazia', 'chi ha idee democratiche', grecismo giunto in italiano attraverso il francese démocratique. Ricordiamo ancora il latinismo astensione 'rinuncia di esercitare il diritto di voto' che è il francese abstension, parola dotta che si rifa al latino tardo abstensiōne $(m)$.

5.3. Nel Settecento incontriamo numerosi francesismi: coalizzare "unire in una coalizione' è il francese coaliser; controrivoluzione 'reazione politica, sociale e militare a una rivoluzione' è il francese contre-révolution; federalismo 'tendenza politica favorevole alla federazione di più Stati' e federalista 'fautore/seguace del federalismo' sono il francese fédéralisme e fédéralist; patriottico 'da patriota', 'ispirato a patriotti- 
smo' e patriottismo 'sentimento di vivo amore verso la patria' sono il francese patriotique e patriotisme; rivoluzionare 'promuovere, realizzare una rivoluzione' e rivoluzionario 'di una rivoluzione', 'della rivoluzione' sono francesismi presi dal francese révolutionner e révolutionnaire.

I grecismi del Settecento sono il verbo transitivo democratizzare 'rendere democratico', 'trasformare in senso democratico' prestito dal greco dēmokratizein, entrato in italiano attraverso il verbo francese démocratiser, il nome oligarca 'membro di un'oligarchia' che deriva dal greco oligárchës e il nome teocrazia 'sistema politico in cui l'autorità politica è esercitata dal potere religioso', che deriva dal greco theokratía, che ha assunto una certa importanza con la rivoluzione francese. L'aggettivo anarchico 'proprio dell'anarchia' è prestito dal greco anarchía 'mancanza di governo'. ${ }^{23} \mathrm{Il}$ nome cosmopolita 'chi riconosce come propria patria il mondo' è prestito dal greco kosmopolitees, grecismo entrato in italiano tramite il francese cosmopolite. Il nome coalizione 'alleanza di persone e partiti per la realizzazione di scopi comuni' è in accezione politica l'inglese coalition; la parola è giunta tramite il francese coalition; l'aggettivo costituzionale 'relativo alla Costituzione' è l'inglese constitutional.

5.4. Nell'Ottocento i francesismi sono ancora più numerosi: alleare 'unire', 'collegare' (1853,) è il francese allier; astensionista è il francese astensionniste; autocratico 'di/da autocrate' è il francese autocratique; autoritario 'che fa valere la propria autorità con fermezza talvolta eccessiva' e autoritarismo 'forma di esagerata autorità esercitata da persone o istituzioni' sono il francese autoritaire e autoritarisme; cesarismo, dal cognome di Gaio Giulio Cesare, 'dittatura politica personale legittimata da un plebiscito e garantita dall'esercito' è il francese césarisme; clericalismo è il francese cléricalisme; comunismo 'dottrina politica, economica e sociale fondata da C. Marx e F. Engels' e comunista sono prestiti dal francese communisme e communiste; cosmopolitismo 'dottrina che considera come un'unica grande patria, senza distinzione di razze e di nazioni' è prestito dal francese cosmopolitisme; militarizzare 'sottoporre a disciplina militare' e militarizzazione 'il militarizzare' sono il francese militariser e militarisation; opportunismo 'comportamento consistente nell'agire senza tenere conto di principi o ideali' e opportunista 'chi agisce con opportunismo', 'opportunistico' sono il francese opportunisme e opportuniste; nazionalismo e nazionalista sono il francese nationalisme e nationaliste; rivoluzionario 'chi/che promuove una rivoluzione' è adattamento del francese révolutionnaire; trasformismo 'metodo di governo adottato da A. Depretis alla fine dell'Ottocento, consistente nell'utilizzare spregiudicatamente persone e gruppi politici in modo da impedire che si formasse una vera opposizione' e trasformista sono adattamenti del francese trasformisme e trasformiste.

23 Alla formazione dell'aggettivo italiano anarchico ha probabilmente contribuito l'esistenza dell'aggettivo francese anarchique. 
Gli anglicismi sono numerosi: assolutismo e assolutista sono l'inglese absolutism e absolutist ${ }^{24}$ cartismo 'movimento politico-sociale che, nella seconda metà del sec. XIX, convogliava le aspirazioni dei lavoratori inglesi verso una maggiore democrazia politica' è l'inglese chartism; l'anglicismo costituzionalismo 'insieme dei principi ispiratori dell'ordinamento supremo dello Stato costituzionale' è l'inglese constitutionalism giunto forse attraverso il francese constitutionalisme; imperialismo e imperialista sono l'inglese imperialism e imperialist; plutocrazia 'predominio politico dei plutocrati o dell'alta finanza e grande industria', plutocrate e plutocratico sono l'inglese plutocra$c y$, plutocrate e plutocratic.

I grecismi sono pochi: i nomi autocrate 'sovrano assoluto', dal greco autocratés e autocrazia 'governo dispotico, tirannico, assoluto', dal greco autocráteia, sono grecismi entrati in italiano attraverso le parole francesi autocrate e autocratie.

Il sostantivo antisemitismo 'atteggiamento, politica ostile nei confronti degli Ebrei' che è il tedesco Antisemitismus e pluralismo 'dottrina politica che difende la pluralità di partiti e sindacati in uno Stato' deriva dal latino plurālis sul modello del tedesco Pluralismus.

5.5. Nel Novecento troviamo molti francesismi: astensionismo è il francese abstentionnisme; cosmopolitismo è il francese cosmopolitisme; egualitario e egualitarismo sono il francese égalitaire e égalitarisme; estremismo e estremista sono il francese extremisme e extremiste, gauchismo e gauchista sono adattamenti di gauscisme e gauchiste; gaullismo e gaullista sono adattamenti del francese gaullisme e gaulliste, ${ }^{25}$ gollista 'proprio di De Gaulle', 'fautore del gollismo' è adattamento del francese gaulliste e gollismo 'tendenza politica che si ispira alle idee del generale francese $\mathrm{Ch}$. De Gaulle' è adattamento del francese gaullisme; militarismo e militarista sono il francese militarisme e militariste; pacifismo e pacifista sono il francese pacifisme e pacifiste; razzismo è il francese racisme e sindacalista è il francese syndicaliste.

Gli anglicismi sono numerosi, meno numerosi dei francesismi: tecnocrate 'uomo politico $o$ alto funzionario la cui autorità si fonda prevalentemente sulla competenza tecnica' e tecnocratico sono l'inglese technocrat e technocratic. ${ }^{26} \mathrm{Gli}$ anglo-americanismi isolazionismo 'condotta di uno Stato che segue la politica di isolamento politico rispetto agli altri Stati' e isolazionista 'fautore dell'isolazionismo' sono l'inglese d'America isolationism e isolationist; populismo 'movimento politico russo di giovani intellettuali della fine del XIX sec., caratterizzato dall'idealizzazione delle masse popolari contadine' e populista 'fautore del populismo' sono l'inglese d'America populism e populist.

Ricordiamo ancora la locuzione inglese mass media 'mezzi di (comunicazione di) massa', composta da mass, dal latino măssa 'massa', e media, altro latinismo, (plurale

24 Alla formazione degli anglicismi assolutismo e assolutista ha probabilmente contribuito l'esistenza delle forme francesi absolutisme e absolutist. 
di medium) 'mezzi'; e il germanismo nazionalsocialismo 'movimento politico nella Germania fra le due guerre' che è calco sul tedesco Nationalsozialismus.

I. forestierismi non adattati sono l'anglicismo apartheid 'politica di segregazione razziale nel Sud Africa' costituito da apart 'separato', che deriva dal francese $a$ part 'a parte', e dal suffisso -heid che denota stato o condizione; $\mathrm{i}$ francesismi gaushisme 'complesso dei movimenti extraparlamentari di sinistra e delle loro ideologie' e gauchiste 'chi/che appartiene politicamente alla sinistra extraparlamentare' che derivano dal francese gauche 'sinistra'; e il russismo perestrojka 'radicale opera di riorganizzazione politico-economica dell'Unione Sovietica avviata da Gorbaciov a partire dal 1985 '.

\section{Conclusione}

Il lessico politico è ricco, innovativo, creativo, moderno; si rinnova sempre; abbonda di neologismi. Tra i suffissi in espansione ci sono i suffissi nominali -ismo, -ista e -zione, i suffissi aggettivali -iano, -ico, -ista e -istico e il suffisso verbale -izzare. Le parole in -ismo di cui è ricco il lessico politico come castrismo, egemonismo, fascismo, gandhismo, integrazionismo, leninismo, regionalismo, socialismo, stalinismo indicano fenomeni politici molto complessi, dottrine politiche controverse, suscettibili di diverse interpretazioni e valutazioni. Godono di una particolare fortuna le formazioni scientifiche; numerose parole composte sono costituite di elementi lessicali latini e greci, cioè di elementi neoclassici. I prefissoidi e i suffissoidi derivati dal latino e dal greco sono numerosi, soprattutto quelli derivati dal greco: auto-, bi-, demo-, mono-, neo-, pluri-, poli-, ultra-, uni-, tele-, video-, -crazia, -logia, -logo.

I1 linguaggio politico ha tendenza a coniare le sigle e le unità lessicali superiori formate da almeno due elementi formativi scritti separatamente.

Tra i fenomeni innovativi bisogna ricordare anche l'accoglimento di gran numero di prestiti, forestierismi e calchi linguistici, soprattutto francesismi e anglicismi, e in particolare l'accoglimento di forestierismi senza adattamento alla morfonologia dell'italiano contemporaneo.

25 Dal nome del generale francese Ch. De Gaulle si ha anche, e più frequentemente, la forma degaullista, nome e aggettivo.

26 Dall'aggettivo tecnocratico si forma l'avverbio di modo tecnocraticamente. 


\section{Bibliografia}

BECCARIA, G.L. (a cura di) 1994 Dizionario di linguistica e di filologia, metrica, retorica, Torino, Einaudi.

Bobbio, N., Matteucci, N., Pasquino, G. (diretto da) 1983 Dizionario di politica, Torino, UTET, seconda edizione interamente riveduta e ampliata.

Cortelazzo, M., Cardinale, U. 1988 Dizionario di parole nuove 1964-1987, Torino, Loescher.

CoRTelazZo, M., Zolli, P. 1999 DELI - Dizionario Etimologico della Lingua Italiana, seconda edizione in volume unico a cura di M. Cortelazzo e M. A. Cortelazzo, Bologna, Zanichelli.

DARDANO, M. 1978 La formazione delle parole nell'taliano di oggi, Roma, Bulzoni.

DARDANO, M. 1986, Il linguaggio dei giornali italiani, Bari, Laterza.

DARDANo, M., Trifone, P. 1997 La nuova grammatica della lingua italiana, Bologna, Zanichelli.

De MAURo, T. 1999 Grande Dizionario Italiano dell'Uso (Gradit), Torino, UTET, in 6 volumi e CD-Rom.

De MaUro, T., Mancini, M. 2001 Dizionario delle parole straniere nella lingua italiana, Garzanti linguistica, Divisione di UTET.

GaRzanti Ed. 1998 Il Grande Dizionario Garzanti della Lingua Italiana, Milano.

D'ORSI, A. (a cura di) 1995 Alla ricerca della politica. Voci per un dizionario, Torino, Bollati Boringhieri.

MARELlo, C. 1996 Le parole dell'italiano, Lessico e dizionari, Bologna, Zanichelli.

Renzi, L., SAlVI, G., CARDINAlETTI, A. (a cura di) 1995 Grande grammatica italiana di consultazione, vol. III, Tipi di frasi, deissi, formazione delle parole, Bologna, Il Mulino.

SERIANNI, L., TRIFONE, P. (a cura di) 1993, 1994 Storia della lingua italiana, 1993 vol. I, I luoghi della codificazione, 1994 vol. II, Scritto e parlato, Torino, Einaudi.

TeKaVČćć, P. 1980 Grammatica storica dell'italiano, Vol. III, Lessico, Bologna, Il Mulino.

TrIFone, P. (a cura di) 1984 Dizionario politico popolare, Ristampa dell'edizione di Torino, 1851, Roma.

VUČETIĆ, Z. 1998 Formazioni scientifiche. Primi materiali, in "Linguistica" XXXVIII, 2, Ljubljana, pp. 167-182.

VUČETIĆ, Z. 2001 La terminologia marinaresca studiata dal punto di vista della formazione delle parole, in "Linguistica" XLI, Ljubljana, pp. 111-128.

VUČETIĆ, Z. 2002 Il linguaggio della giurisprudenza dal punto di vista della formazione delle parole. Orientamenti e problemi lessicologici, in "Linguistica" XLII, Ljubljana, pp. 65-80.

Zingarelli, N. 2002 Vocabolario della Lingua Italiana, Bologna, Zanichelli. 


\section{POLITIČNI JEZIK. POLITIČNO BESEDJE}

Besedje je v političnem jeziku nujno bogato, dovzetno za novosti, močno kreativno. Polno je neologizmov. Prispevek še posebej poudarja izrabo samostalniških pripon, kot -ismo, -ista, -zione, pridevniških kot -iano, -ico, -ista, -istico, glagolskih kot -izzare.

Te novotvorbe so velikokrat razumljene različno, ker skušajo pač izražati različne ideološke poglede. $\mathrm{V}$ leksemskem delu najdemo zlasti grške in latinske besedne prvine, seveda tudi lastna imena. Grški ali latinski vir pa imajo razen pripon, kot -crazia, -logo, tudi predpone, npr. auto-, demo-, mono-, poli-, ultra-ipd.

Značilnost italijanskega političnega jezika je in je zmeraj bila prevzemanje tujih leksikalnih prvin, nekdaj predvsem iz francoščine, v modernih časih iz angleščine. 